\title{
Recent advances in magnetic nanoparticle-based microfluidic devices for the pretreatment of pathogenic bacteria
}

\author{
Hyunsoo Han ${ }^{1} \cdot$ Bokyeong Sohn ${ }^{1} \cdot$ Jihun Choi $^{1} \cdot$ Sangmin Jeon $^{1}$ (I)
}

Received: 21 June 2021 / Revised: 6 August 2021 / Accepted: 8 August 2021 / Published online: 19 August 2021

(c) Korean Society of Medical and Biological Engineering 2021

\begin{abstract}
Rapid and sensitive detection of pathogenic bacteria in various samples, including food and drinking water, is important to prevent bacterial diseases. Most bacterial solutions contain only a small number of bacteria in complex matrices with impurities; hence, pretreatment is necessary to separate and concentrate target bacteria before sensing. Among various pretreatment methods, iron oxide magnetic nanoparticle (MNP)-based pretreatment has drawn attention owing to the unique properties of MNP, such as high magnetic susceptibility, superparamagnetism, and biocompatibility. After target bacteria are captured by recognition molecule-functionalized MNPs, bacteria-MNP complexes can be easily separated and enriched by applying an external magnetic field. Various devices, such as optical, electrochemical, and magnetoresistance sensors, can be used to detect target bacteria, and their detection principles have been discussed in numerous review papers. Herein, we focus on recent research advances and challenges in magnetic pretreatment of pathogenic bacteria using microfluidic devices, which offer the advantages of process automation and miniaturization.
\end{abstract}

Keywords Magnetic nanoparticles $\cdot$ Pathogenic bacteria $\cdot$ Microfluidic $\cdot$ Pretreatment

\section{Introduction}

Pathogenic bacteria pose serious problems worldwide as they cause diseases, such as tuberculosis, pneumonia, sepsis, and food poisoning [1]. Among them, food poisoning is caused by the consumption of food contaminated with pathogenic bacteria. According to the World Health Organization, eating contaminated food causes illness in $\sim 600$ million people and 420,000 deaths every year [2]. Thus, sensitive methods for detecting pathogenic bacteria in food are important to ensure food safety. A typical method for detecting pathogenic bacteria is culturing. This method is used as a golden standard because it can detect pathogenic bacteria sensitively and specifically. However, culturing of a sample takes several days, which hinders the real-time application of the method [3, 4]. Various detection methods, such as adenosine triphosphate (ATP) luminescence, enzyme-linked immunosorbent assay, and polymerase chain reaction, have

Sangmin Jeon

jeons@ postech.ac.kr

1 Department of Chemical Engineering, Pohang University of Science and Technology (POSTECH), Pohang, Gyeongbuk, Republic of Korea attracted attention because bacteria can be detected in a few hours [5-8].

Although these methods are sensitive and require only a small sample volume, they are not suitable for detecting pathogenic bacteria in large samples of foods and drinking water. Since only trace amounts of bacteria are present, it can produce a false negative signal when a small sample volume is used for detection. In addition, the presence of various impurities in samples can interfere with the detection and reduce sensitivity. To overcome these limitations, a pretreatment process that separates and concentrates target bacteria is required. Filtration and centrifugation are the conventional methods for pretreating bacterial solutions [9-12]. These methods are used extensively in many areas because they are simple, rapid, and inexpensive. However, filtration and centrifugation involve several steps of target bacteria concentration and washing, leading to bacterial damage and sample loss. In addition, these methods are difficult to integrate with detection methods, limiting the automation of the entire process and manufacturing of portable devices. Because of these limitations, magnetic nanoparticle (MNP)based pretreatment methods have attracted considerable attention as promising alternatives. 
Magnetic separation mainly uses iron oxide $\left(\mathrm{Fe}_{3} \mathrm{O}_{4}\right)$ MNPs to separate and enrich targets owing to their unique properties, including high magnetic susceptibility, superparamagnetism, and biocompatibility [13, 14]. With superparamagnetism, MNPs can be separated using an external magnetic field and redispersed without the aggregation of particles $[15,16]$. The surface of MNPs can be easily functionalized through surface treatments, such as carbodiimide hydrochloride/N-hydroxy succinimide reaction and silane treatment $[17,18]$. Then, recognition molecules, such as polymers, aptamers, and antibodies, are fixed on treated surfaces [19-23]. When recognition moleculefunctionalized MNPs are bound to bacteria, the bacteria-MNP complexes can be separated using permanent magnets with minimal damage to the bacteria [24-27]. However, conventional MNP-based separation methods require multiple steps of mixing and transfer, such as binding, washing, and enrichment. Besides, it takes from 30 min to several hours to capture target bacteria as the sample volume increases.

These problems can be addressed using microfluidic devices that offer advantages such as process automation, cost-effectiveness, and miniaturization [28-30]. Through microfluidic-based automation and miniaturization, portable devices that can be used by people lacking expertise can be fabricated. The synergistic effect arising from combining MNPs with microfluidics provides high performance in pretreatment and detection compared to conventional methods. The limitation of microfluidics involving the difficulty to handle large volumes of samples can also be addressed by creating virtual filters comprising MNPs [31-33]. A virtual filter is formed by aligning recognition molecule-functionalized MNPs in the direction of an external magnetic field, which allows liquid and impurities to pass through but captures target bacteria. As a sample solution containing bacteria is injected, the MNPs and bacteria bind with a high probability, and the bacteria are concentrated inside the channel. However, both bacteria-MNP complexes and free MNPs are present in the virtual filter. Because the presence of free MNPs reduces detection sensitivity, efforts have been made to separate bacteria-MNP complexes from free MNPs using hydrodynamic forces or porous membranes [34-36]. In particular, selective filtration and lateral flow immunomagnetic assay (LFA) using MNPs and porous membranes, allow for the simultaneous isolation and detection of target bacteria $[37,38]$. The presence of numerous interconnected microchannels in the membrane allows sample solutions to pass through the microchannels at relatively high flow rates, resulting in rapid binding of target bacteria in the sample solution to the recognition molecules functionalized on the channel surface. In this mini-review, we outline recent trends in the magnetic separation and enrichment of pathogenic bacteria inside fluidic channels.

\section{Magnetic separation and enrichment of pathogenic bacteria using MNPs and microfluidics}

Separation and enrichment of bacteria are important to detect low concentrations of pathogenic bacteria in large samples. For selective capturing of target bacteria, the surfaces of MNPs are functionalized with recognition molecules, such as antibodies and aptamers. Using detection methods that do not require a selective separation, such as polymerase chain reaction, polyelectrolytes capable of electrostatically binding to target bacteria can be coated on the MNPs surface $[15,39]$. For the separation of bacteria-MNP complexes from sample solutions by applying an external magnetic field, a conventional method uses a multiple-step process. After MNPs are bound to target bacteria in a chamber, bacteria-MNP complexes are transferred for washing and concentration. On the contrary, a recent trend is to perform binding and separation in microfluidic channels. This chapter presents recent trends in pretreatment methods using MNPs and microfluidics.

\subsection{Conventional methods for separating pathogenic bacteria in microfluidic channels}

By applying an external magnetic field, bacteria-MNP complexes can be easily separated in microfluidic channels based on magnetophoresis [40-45]. When a solution containing bacteria-MNP complexes is injected into a fluidic channel under a magnetic field, the complexes are attracted to the wall of the channel close to the external magnetic field, whereas the solution containing impurities flows along the center of the fluidic channel; thus, the bacteria-MNP complex is separated from impurities. Although this magnetophoresis-based separation is simple, the efficiency of separation is limited. Since the intensity of the magnetic field is inversely proportional to the distance, MNPs do not receive a sufficient magnetic force, as the width of the channel increases to accommodate high flow rates.

To address this problem, Kang et al. fabricated a microfluidic device with a slanted ridge array (Fig. 1a) [43]. The array induces advective rotational flows that move MNPs toward the channel wall where magnetic forces are high, separating $92 \%$ of Escherichia coli (E. coli)-bound with MNPs from undiluted whole blood at a flow rate of $0.6 \mathrm{~mL} / \mathrm{h}$. Although the separation performance of the microfluidic device with a slanted ridge array is better than that of conventional microfluidic devices of similar dimensions, it is not suitable for handling large sample solutions due to its too low flow rates. 
(a)

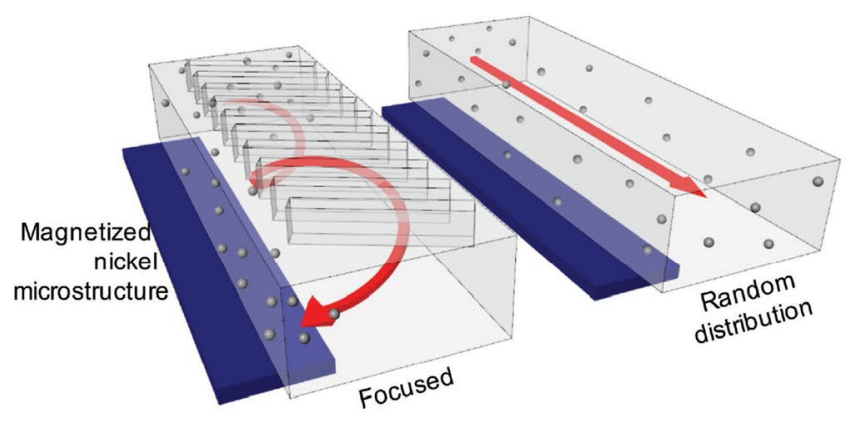

Fig. 1 Separation of bacteria using magnetophoresis: a Microfluidicmagnetic separation device integrated with a slanted ridge array, which induces advective rotational flows [43]. b Magnetic separation

Yang et al. increased the flow rate by increasing the length of the region where magnetophoresis occurred [44]. Figure $1 \mathrm{~b}$ shows a schematic of a three-dimensional device in which a commercial plastic tube is wrapped around a permanent magnet. When a solution containing bacteria-MNP complexes is injected into the microfluidic channel, the solution flows around the magnet, and the complexes are separated via magnetophoresis. Due to the increase in the magnetophoresis distance, bacteria-MNP complexes could be separated at a flow rate of $0.67 \mathrm{~mL} /$ min with a separation efficiency of $92 \%$. In addition, the device enabled the continuous separation of bacteria with a concentration factor of 110 .

Interestingly, different bacterial types can be separated and concentrated simultaneously using different magnetic materials with different magnetization [45]. Figure 2 shows a schematic of a device that separates the weakly magnetized nanoparticle-bound Salmonella from the strongly magnetized nanoparticle-bound $E$. coli. Each MNP is functionalized (b)

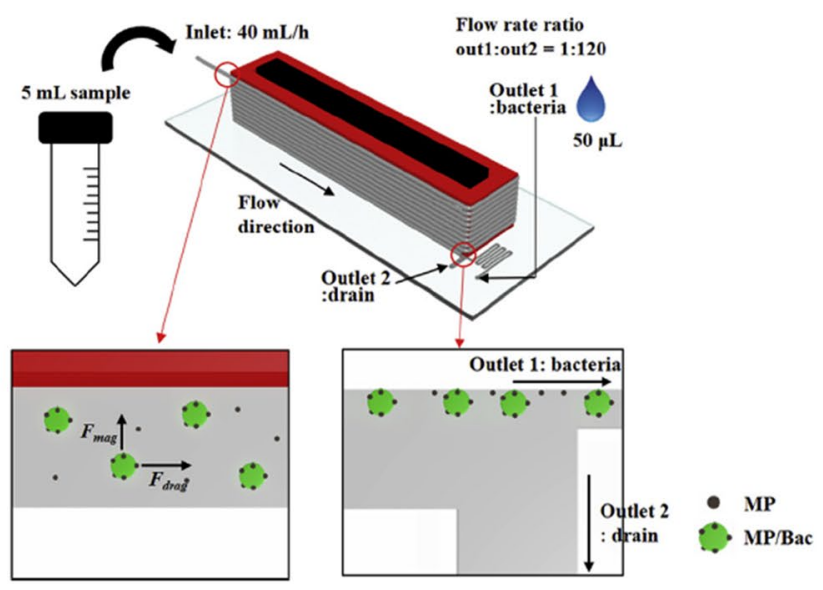

device composed of a permanent magnet wrapped with a plastic tube. The long tube allows continuous separation of bacteria with high separation efficiency at a higher flow rate [44]

with a different antibody to selectively capture the target bacteria. When a sample solution is injected with a sheath flow, the strongly magnetized nanoparticle-bound $E$. coli is attracted toward the permanent magnet and is collected from the upper outlet, and separates from the weakly magnetized nanoparticle-bound Salmonella, which is collected from the lower outlet.

\subsection{Rapid separation of pathogenic bacteria using virtual filters comprised of MNPs}

Magnetic separation is simple and fast. However, the binding of MNPs to target bacteria takes time as it occurs through Brownian collisions of MNPs and target bacteria in solution. Typical binding times (i.e., capture times) range from 30 min to several hours, depending on the concentration of MNPs used and the sample volume. It is the most time-consuming process in the pretreatment process. In contrast, binding in LFA takes only a few minutes [46]. Targets
Fig. 2 Separation of Escherichia coli and Salmonella typhimurium by strongly and weakly magnetized MNPs, respectively [45]

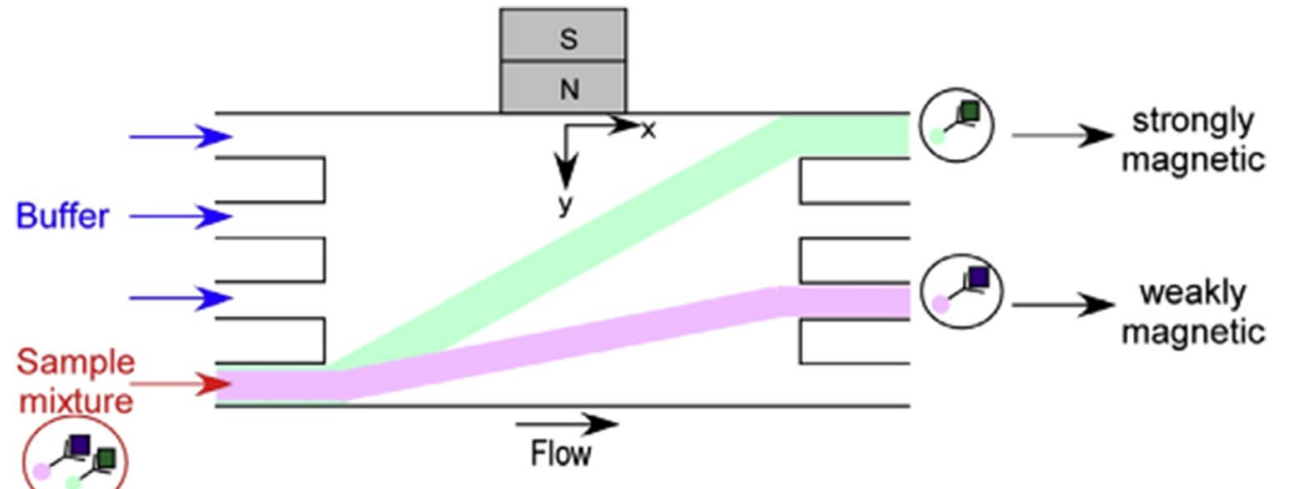

E. coli 0157 bound Hyglos bead Dyna bead
S. typhimurium bound 
rapidly bind to antibodies immobilized at the test line during the solution flow owing to the antibody-immobilized membrane, which acts as a filter and captures target bacteria in the flowing solution. Similarly, if MNPs are uniformly dispersed and block the cross section of the fluidic channel, the MNPs can act as a filter. When a permanent magnet is placed near the channel containing an MNP solution, the MNPs are aligned along the external magnetic field lines to create a wall (i.e., a virtual filter). Unlike conventional membrane filters, virtual filters comprising MNPs are flexible, allowing the penetration of large particles if they do not bind to MNPs. As a result, only target bacteria are captured by the virtual filter when a sample solution containing target bacteria is injected into the channel. The virtual filter method is the most efficient regarding the capture time and separation performance.

However, creating a uniform and stable virtual filter, particularly under high flow rates, is not straightforward. When a permanent magnet is placed close to a channel containing an MNP solution, the MNPs are attracted to the inner surface of the channel near the magnet without blocking the cross section of the fluidic channel; thus, reducing the capture efficiency. When a permanent magnet is placed away from the channel, in contrast, MNPs can form a uniform wall across the channel, but the wall is easily destroyed by solutions flowing at high flow rates because the magnetic force is not strong enough to retain the MNPs. Efforts have been made to create a stable and uniform virtual filter across the channel $[32,47,48]$. Figure 3 a shows a schematic of a fluidic device with sawtooth-shaped iron foils placed on a permanent magnet [48]. The laminated sawtooth-shaped iron foils generate dot-array high gradient magnetic fields and focus the magnetic field at the tips of the sawtooth. Thus, MNPs inside a fluidic channel align along the magnetic field lines at the tips, forming a chain-like wall, thereby increasing the effective collision between target bacteria and MNPs. The bacterial capture efficiency is about $80 \%$, which is twice as high as that in the absence of the iron foil at $50 \mu \mathrm{L} / \mathrm{min}$. Figure $3 b$ shows a schematic of the double-layer capillarybased immunomagnetic separation process [32]. The inner capillary is filled with iron balls magnetized by a permanent magnet placed below the outer capillary. This device generates a magnetic field strength of $0.76 \mathrm{~T}$ and a magnetic gradient of $100 \mathrm{~T} / \mathrm{m}$. The capture efficiency was $\sim 80 \%$ when $1 \mathrm{~mL}$ of an E.coli solution was recycled for $45 \mathrm{~min}$.

Most studies on magnetic separation use flat permanent magnets, which generate a magnetic field perpendicular to the solution flow. However, the perpendicular magnetic field is inefficient to form a stable virtual filter because a uniform filter is formed only when a permanent magnet is somewhat away from the channel, which weakens the magnetic force to retain MNPs at high flow rates. Lee et al. addressed this problem by generating a magnetic field in the opposite direction of the solution flow. Figure 4a shows a schematic of the device in which a glass channel is wrapped with copper tape [31]. The glass channel is placed between two permanent magnets, which create a perpendicular magnetic field and magnetize MNPs. When a sample solution is injected into the channel, the drag force moves the MNPs away from their original positions. According to Lenz's law, the flow-induced movement of MNPs in a conductive channel under an external magnetic field creates a magnetic force in an opposite direction to the solution flow, increasing the stability of the virtual filter at high flow rates. The separation efficiency of target bacteria is $85 \%$ at a flow rate of $1 \mathrm{~mL} / \mathrm{min}$.

Another challenge in using a virtual filter is to ensure its uniformity. MNPs that make up a virtual filter should be dispersed uniformly and densely to efficiently capture target bacteria. However, it is not easy to obtain a uniform dispersion of MNPs throughout the channel using flat permanent magnets. Figure $4 \mathrm{~b}$ shows a photo, simulated magnetic field lines, and microscopy image of MNPs inside a Halbach ring comprising 12 curved trapezoidal magnets [39]. Each magnet is positioned such that it has a specific magnetic orientation, which varies by $60^{\circ}$ from the adjacent magnet. In this arrangement, the uniform magnetic field lines are formed inside the ring, and the bacterial capture efficiency is $\sim 90 \%$ at a flow rate of $5 \mathrm{~mL} / \mathrm{min}$.

\section{Isolation of target bacteria-MNP complexes from free MNPs}

After bacteria-MNP complexes are separated from a sample solution, various devices, such as optical, electrochemical, and magnetoresistance sensors, can be employed to detect target bacteria [49-51]. Detailed detection methods have already been discussed in numerous review papers [52-55]. The most sensitive detection method employs optical probes, such as gold nanoparticles, fluorescent dyes, and quantum dots [37, 56, 57]. However, both bacteria-MNP complexes and free MNPs are present in the solution after magnetic separation because the concentration of MNPs used is much higher than the concentration of target bacteria. Since the presence of free MNPs interferes with optical measurements and reduces detection sensitivity, efforts have been made to separate bacteria-MNP complexes from free MNPs. Once bacteria-MNP complexes are isolated from free MNPs, the bacterial concentration can be determined using various simple methods, such as light absorption or colorimetry $[34,58]$. Herein, the isolation of bacteria-MNP complexes from free MNPs is discussed. 
(a)
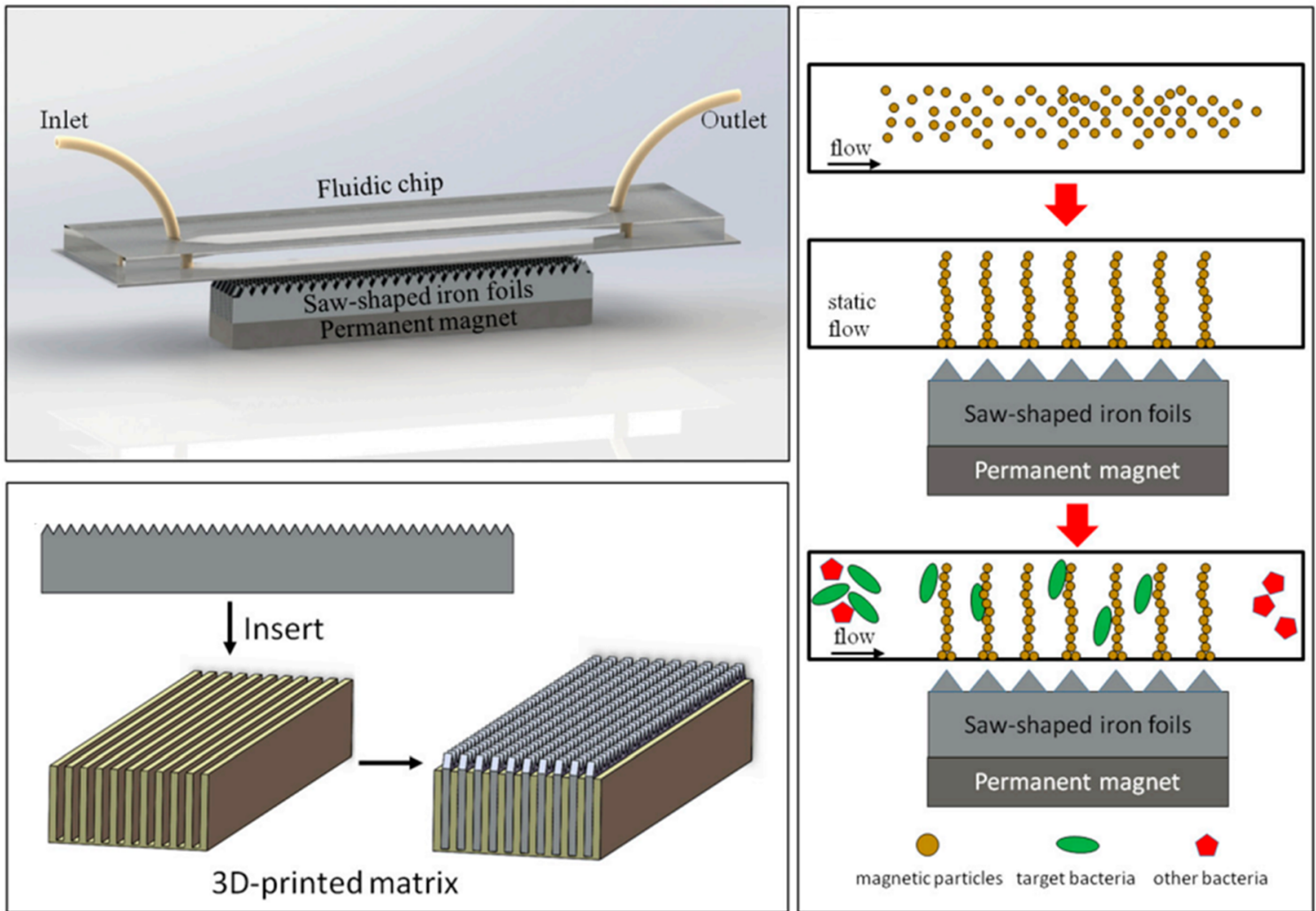

(b)

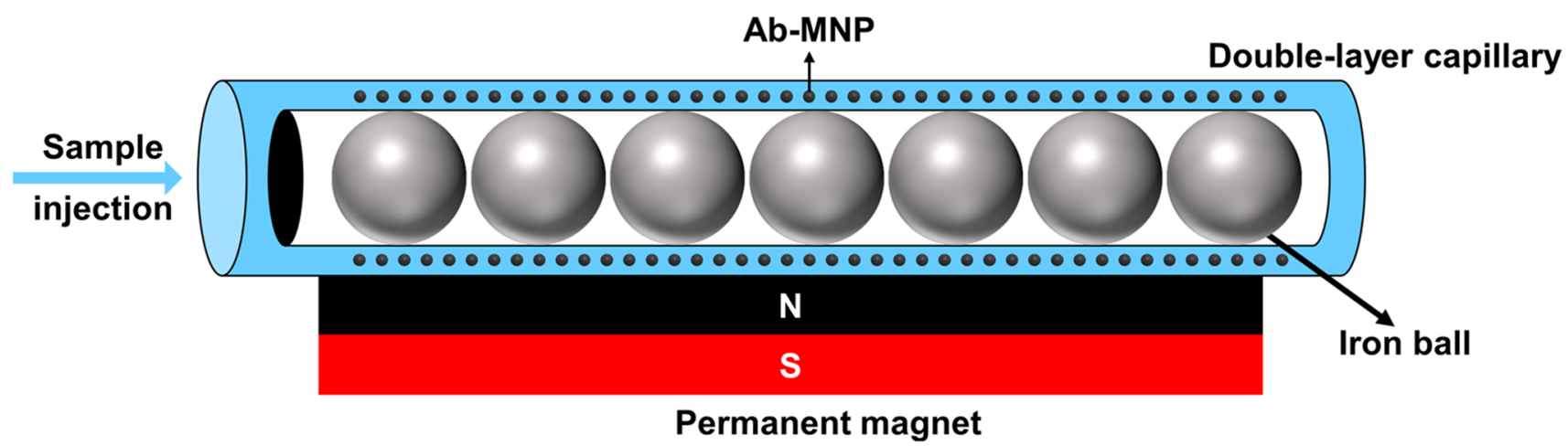

Fig. 3 Separation of bacteria using virtual filters comprising MNPs. A virtual filter is formed using a sawtooth-shaped iron foils on a permanent magnet [48] and $\mathbf{b}$ a double-layer capillary with iron balls [32]

\subsection{Isolation of bacteria-MNP complexes from free MNPs using hydrodynamic forces}

Magnetophoresis-based separation can isolate both free MNPs and bacteria-MNP complexes from sample solutions. Since the concentration of MNPs is usually much higher than that of target bacteria, significant amounts of free
MNPs are present in the solution, increasing background noise and decreasing detection sensitivity. Various studies have been conducted to separate bacteria-MNP complexes from free MNPs based on hydrodynamic forces [34, 35, $59,60]$. Figure 5a shows a schematic of the separation of bacteria-MNPs and free MNPs using elastic and inertial lift forces [34]. After adding MNPs to a sample solution 
Fig. 4 Increase in the stability and uniformity of a virtual filter under high flow rates using a magnetic force in the opposite direction of the solution flow [31]. b Halbach ring comprising 12 magnets [39]; the black arrows indicate the magnetic orientation and linear magnetic field lines are formed inside the Halbach ring. The cross-sectional image shows that MNPs are uniformly dispersed inside the channel

(a)

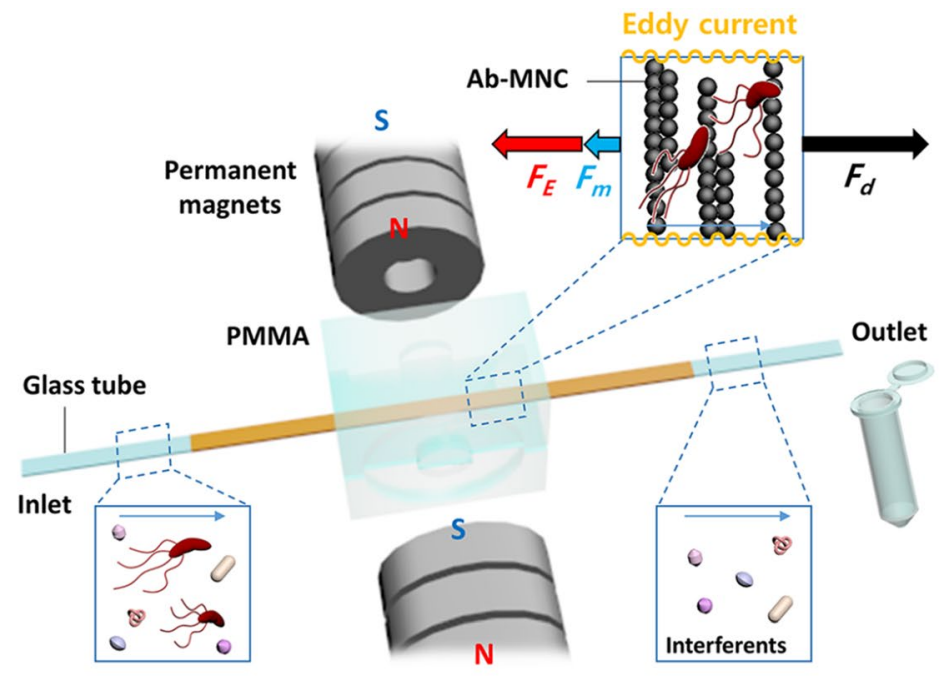

(b)
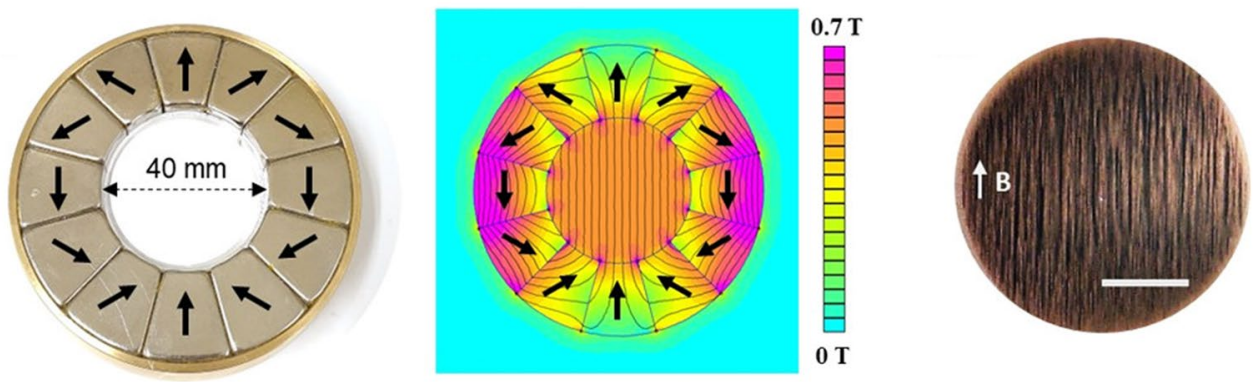

(a)

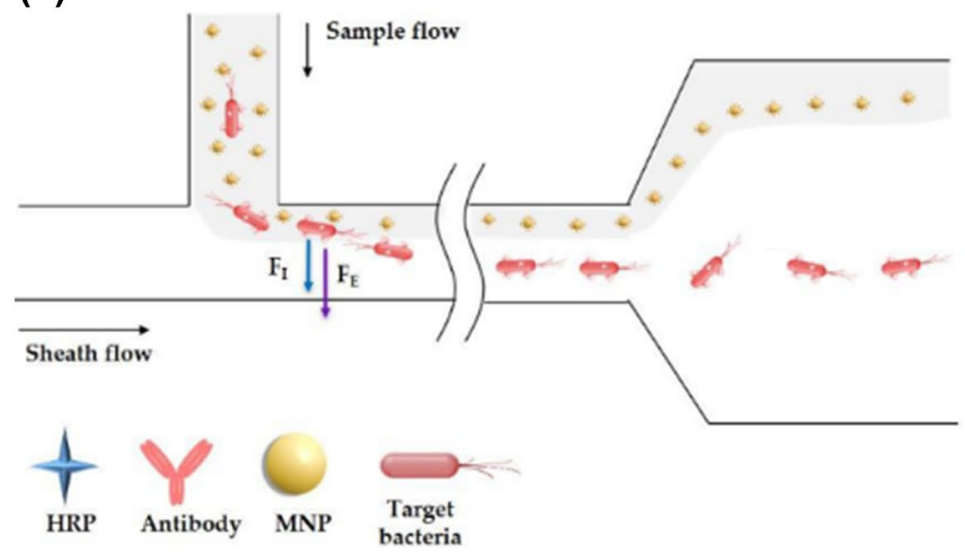

(b)

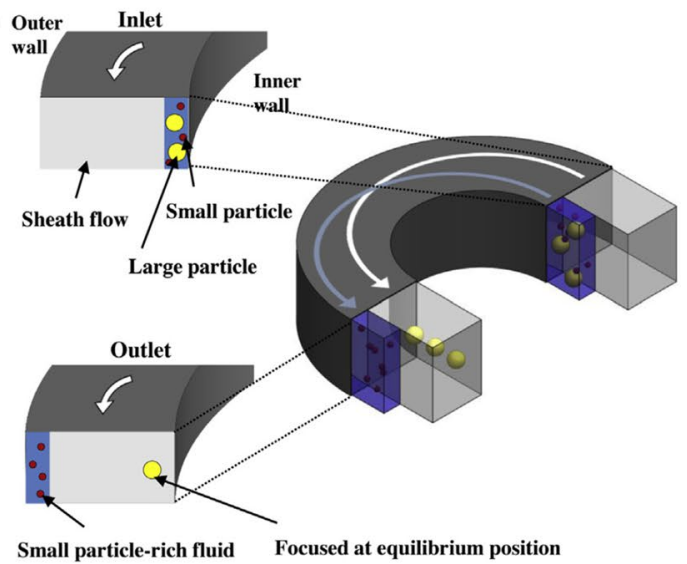

Fig. 5 Separation of bacteria-MNP complexes from free MNPs using hydrodynamic forces: a Elastic and inertial lift forces [34]. b Dean flow and inertial force inside a spiral channel [35]

containing target bacteria and polyvinylpyrrolidone (PVP), the sample solution is injected into the top inlet of the channel, and a PVP solution is injected into the side inlet as a sheath flow. The sample solution flow is squeezed by the sheath flow, inducing elastic and inertial lift forces. The elastic lift force moves the particles to the center of the channel at a low shear rate, and the inertial lift force moves them away from the sidewall. The elastic and inertial lift forces are proportional to the radius of the third and sixth power, respectively, and the size variation causes a difference in 
the forces. Thus, bacteria-MNP complexes in the sample solution can be moved to the center of the channel, but the free MNPs are separated by being moved along the wall of the channel.

Another hydrodynamic device using spiral microchannels has been reported [35, 60]. Figure $5 \mathrm{~b}$ shows a schematic of the device for separating bacteria-MNPs from free MNPs based on Dean flow and inertial force [35]. The device has two inlets and multiple outlets. The sample solution is injected into one inlet and the sheath flow is injected into the other. When the two fluids pass through a curved channel, Dean flows are formed, resulting in a Dean force, which is proportional to the hydrodynamic diameter of the particle. The bacteria-MNP complexes and free MNPs differ approximately tenfold in size. Passing through the device, bacteria-MNP complexes migrate to the inner side of the channel wall and free MNPs to the outer side, effectively separating the bacteria-MNP complexes. Fabricating the device on a flat substrate is straightforward but challenged by the variation of the curvature of the 2D spiral channel. Lee et al. addressed this problem by fabricating a helical microchannel around a cylindrical chamber using a 3D printer, and they separated bacteria-MNP complexes from free MNPs at a flow rate of $5 \mathrm{~mL} / \mathrm{min}[60]$.

\subsection{Isolation of bacteria-MNP complexes from free MNPs using magnetophoretic chromatography}

Chromatography is a method for separating mixtures dissolved in a fluid, and it has wide applications. Conventional chromatography is based on the difference in the affinity of mixture components for a solid stationary phase, whereas magnetophoretic chromatography is based on the difference in the settling velocity of MNPs of different sizes under an external magnetic field [61]. Figures 6a, b show schematic illustrations of magnetophoretic chromatography before and after magnetic separation, respectively. After a mixture of bacteria-MNP complexes and free MNPs is sucked into a pipette tip, a viscous polyethylene oxide (PEO) solution is added, forming an unmixed liquid layer. The surface tension of a liquid increases with the viscosity of the liquid. When a permanent magnet is placed below the pipette tip, both bacteria-MNP complexes and free MNPs experience magnetic, drag, and gravitational forces, as they are attracted toward the magnet. The magnetic force is proportional to the apparent volume of MNPs, indicating that the settling velocity of bacteria-MNP complexes is higher than that of free MNPs. As a result, only bacteria-MNP complexes with a higher settling velocity can overcome the surface tension and reach the bottom of the pipette tip, and the target bacteria can be detected with the eyes. The detection limit of magnetophoretic chromatography for $10 \mathrm{~min}$ is $100 \mathrm{cfu} / \mathrm{mL}$.

\subsection{Isolation of bacteria-MNP complexes from free MNPs using porous membranes}

Membrane filtration is a physical separation method based on differences in the size of molecules. Since membrane filtration is economical and does not require specialized skills, it has been employed in various fields [62, 63]. Conventional membrane filtration methods are not target-specific because separation is achieved only by size differences. However, selective filtration to separate target bacteria can be achieved using target-specific recognition molecule-functionalized MNPs and membranes of appropriate pore sizes [36, 64, 65]. Figure 7 shows a schematic of the selective filtration method for detecting Listeria monocytogenes. After capturing Listeria using monoclonal antibody-functionalized MNPs, the solution is passed through a nitrocellulose membrane having a pore size of $1.2 \mu \mathrm{m}$ under vacuum pressure. Relatively small free MNPs ( 100 nm) are released from the membrane, and large bacteria-MNP complexes remain on the membrane, allowing the detection of target bacteria by color change. Although this method exhibits high sensitivity and short assay time, it requires an external power source for (a)

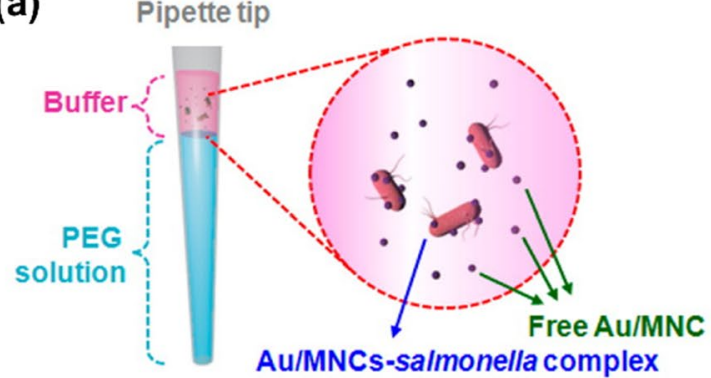

(b)

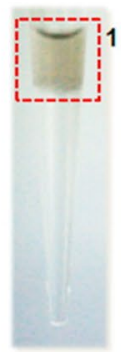

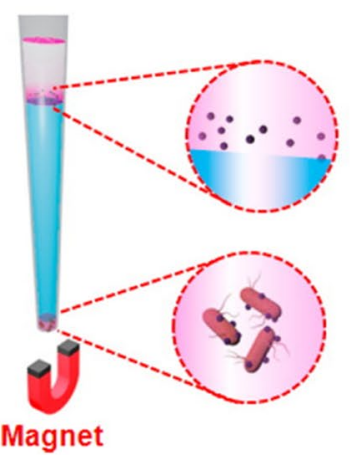

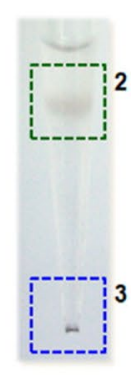

Fig. 6 Schematics and the corresponding photos of magnetophoretic chromatography in a precision pipette tip a before and $\mathbf{b}$ after magnetophoretic chromatography [61] 


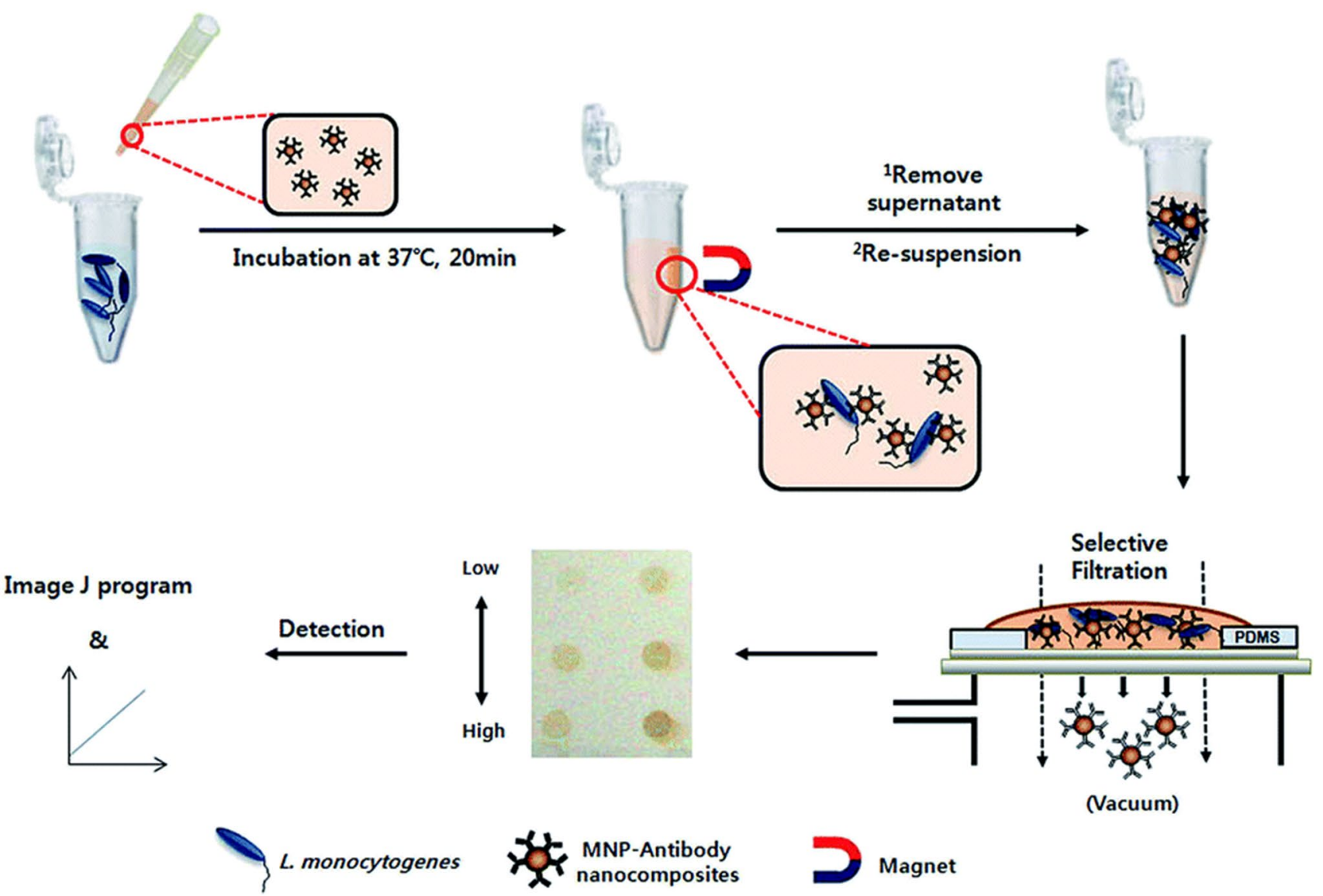

Fig. 7 Schematic of the selective filtration method based on MNPs and nitrocellulose membranes under vacuum pressure. Large bacteria-MNP complexes are separated from free MNPs using a nitrocel-

vacuum filtration, and its detection sensitivity is degraded by the lateral spread of solutions.

In contrast to selective filtration, LFA does not require an external power source and does not depend on size differences. It separates bacteria-MNP complexes from free MNPs using a membrane immobilized with recognition molecules that can specifically bind to target bacteria [38, $66,67]$. The membrane used in LFA contains numerous interconnected microchannels that allow a sample solution to flow via capillary action. Bacteria-MNP complexes are captured in a test line where target bacteria-specific recognition molecules are immobilized, and free MNPs are captured in a control line where MNP-binding molecules are immobilized. Color change in the test line, which depends on the concentration of bacteria-MNP complexes, is used for detecting target bacteria.

However, this approach requires a pretreatment process, making it less convenient to use. The poor sensitivity of LFA without an enriching step results mainly from the limited interaction between the target bacteria and the recognition molecules immobilized in the test line. Because lulose membrane with a pore size of $1.2 \mu \mathrm{m}$. The concentration of Listeria monocytogenes is determined by the membrane color change due to the filtered complexes [64]

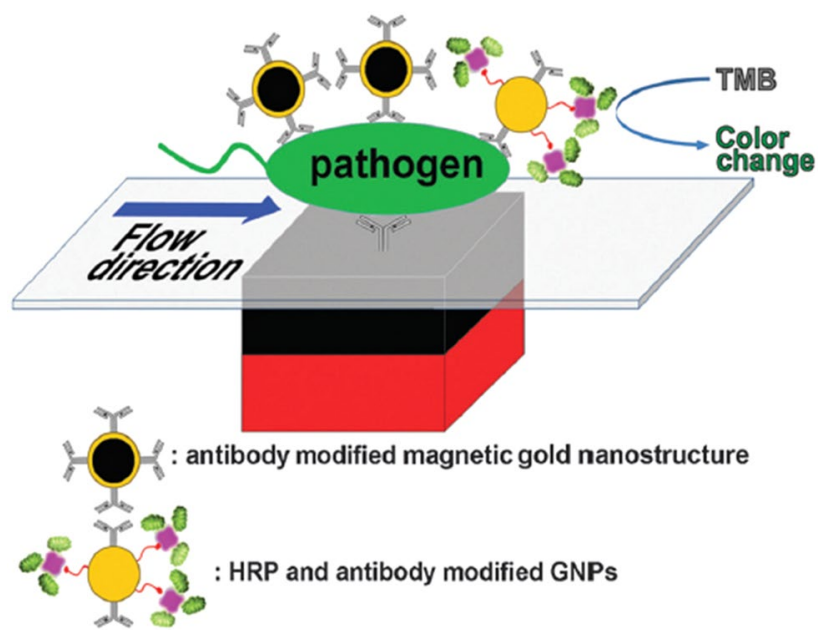

Fig. 8 Schematic of the magnetic focus lateral flow immunomagnetic assay (LFA) using antibody-functionalized MNPs and antibody/ horseradish peroxidase (HRP)-functionalized gold nanoparticles [66]. By placing a permanent magnet under the LFA strip, the movement of the bacteria-MNP complexes is slowed, thereby increasing the binding reaction time between the complex and the antibody immobilized on the membrane 
the microchannels inside the membrane are much larger than the target bacteria-MNP complexes, the sample solution containing the complexes passes through the membrane at a high flow rate, leaving some bacteria uncaptured. Ren et al. developed a magnetic focus LFA (mLFA) method by placing a permanent magnet under an LFA strip to slow down the movement of MNPs. Figure 8 shows a schematic of mLFA using two types of nanoparticles: antibody-functionalized MNPs and antibody/horseradish peroxidase (HRP)-functionalized gold nanoparticles. When the LFA strip is immersed in a solution containing target bacteria, MNPs, and gold nanoparticles, the MNPbound target bacteria flows through the membrane and slows down in the detection zone due to the magnetic field, increasing the binding reaction time between the complex and the antibody immobilized on the membrane. After adding the TMB solution to the membrane, the oxidation of TMB by HRP induces a color change, further increasing the detection sensitivity. The detection limits for E. coli O157:H7 and Salmonella typhimurium in mLFA were 23 and $\sim 17 \mathrm{cfu} / \mathrm{mL}$, respectively [66].

\section{Conclusions and future outlooks}

In this mini-review, we present an overview of bacterial separation methods based on MNPs and fluidic channels. Most review papers reported to date discussed the detection techniques of target bacteria considering sensitivity and selectivity. However, in some applications, such as detecting pathogenic bacteria in food and drinking water, a pretreatment step for isolating and concentrating target bacteria is essential for detection. When only trace amounts of bacteria are present in real samples, a false negative signal can be produced if a small sample volume is used for detection. This can be prevented by adopting an MNP-based pretreatment process. Combining MNPs and microfluidics offers great advantages, such as process automation, cost-effectiveness, and miniaturization, but presents difficulties in processing large amounts of samples in a short time. Virtual filters comprising MNPs have attracted much attention as a promising solution to these problems. Unlike conventional membrane filters, flexible virtual filters selectively capture target bacteria while passing large particles. Various studies are conducted to maintain the stability and uniformity of virtual filters at high flow rates. The remaining challenges in pretreatment with MNPs are to develop methods to distinguish between dead and live bacteria and to isolate target viruses from various samples. In particular, in the aftermath of the COVID-19 pandemic, there is an urgent need to develop MNP-based methods for isolating target viruses that enable sensitive detection.
Acknowledgements This research was supported by the National Research Foundation of Korea (NRF) funded by the Ministry of Science and ICT (NRF-2019R1A2C1084182).

\section{Declaration}

Conflict of interest The authors declare that they have no conflict of interest.

Ethical statement This article does not contain studies with human participants or animals.

\section{References}

1. Ahmed A, Rushworth JV, Hirst NA, Millner PA. Biosensors for whole-cell bacterial detection. Clin Microbiol Rev. 2014;27(3):631-46.

2. World Health Organization (WHO). WHO estimates of the global burden of foodborne diseases. Technical report. Geneva: WHO; 2016.

3. Bartie C, Venter SN, Nel LH. Identification methods for Legionella from environmental samples. Water Res. 2003;37(6):1362-70.

4. Pissuwan D, Gazzana C, Mongkolsuk S, Cortie MB. Single and multiple detections of foodborne pathogens by gold nanoparticle assays. WIREs Nanomed Nanobiotechnol. 2020;12(1):e1584.

5. Fratamico PM. Comparison of culture, polymerase chain reaction (PCR), TaqMan Salmonella, and Transia Card Salmonella assays for detection of Salmonella spp. in naturally-contaminated ground chicken, ground turkey, and ground beef. Mol Cell Probes. 2003; 17(5):215-21.

6. Yang S, Rothman RE. PCR-based diagnostics for infectious diseases: uses, limitations, and future applications in acute-care settings. Lancet Infect Dis. 2004;4(6):337-48.

7. Arroyo MG, Ferreira AM, Frota OP, et al. Effectiveness of ATP bioluminescence assay for presumptive identification of microorganisms in hospital water sources. BMC Infect Dis. 2017;17(1):458.

8. Shih C-M, Chang C-L, Hsu M-Y, et al. Paper-based ELISA to rapidly detect Escherichia coli. Talanta. 2015;145:2-5.

9. Kim J-H, Oh S-W. Optimization of bacterial concentration by filtration for rapid detection of foodborne Escherichia coli $\mathrm{O} 157: \mathrm{H} 7$ using real-time PCR without microbial culture enrichment. J Food Sci. 2019;84(11):3241-5.

10. Wolffs PFG, Glencross K, Thibaudeau R, Griffiths MW. Direct Quantitation and detection of salmonellae in biological samples without enrichment, using two-step filtration and real-time PCR. Appl Environ Microbiol. 2006;72(6):3896-900.

11. Wu X, Han C, Chen J, Huang Y-W, Zhao Y. Rapid detection of pathogenic bacteria from fresh produce by filtration and surfaceenhanced Raman spectroscopy. JOM. 2016;68(4):1156-62.

12. Tsuru A, Setoguchi T, Kawabata N, et al. Enrichment of bacteria samples by centrifugation improves the diagnosis of orthopaedicsrelated infections via real-time PCR amplification of the bacterial methicillin-resistance gene. BMC Res Notes. 2015;8(1):288.

13. Haun JB, Yoon T-J, Lee H, Weissleder R. Magnetic nanoparticle biosensors. WIREs Nanomed Nanobiotechnol. 2010;2(3):291-304.

14. Shabestari Khiabani S, Farshbaf M, Akbarzadeh A, Davaran S. Magnetic nanoparticles: preparation methods, applications in cancer diagnosis and cancer therapy. Artif Cells Nanomed Biotechnol. 2017;45(1):6-17. 
15. Li Z, Ma J, Ruan J, Zhuang X. Using positively charged magnetic nanoparticles to capture bacteria at ultralow concentration. Nanoscale Res Lett. 2019;14(1):195.

16. Lim M-C, Park JY, Park K, Ok G, Jang H-J, Choi S-W. An automated system for separation and concentration of food-borne pathogens using immunomagnetic separation. Food Control. 2017;73:1541-7.

17. Liu S, Yu B, Wang S, Shen Y, Cong H. Preparation, surface functionalization and application of $\mathrm{Fe} 3 \mathrm{O} 4$ magnetic nanoparticles. Adv Colloid Interface Sci. 2020;281:102165.

18. Wang L, Lin J. Recent advances on magnetic nanobead based biosensors: from separation to detection. TrAC Trends Anal Chem. 2020;128:115915.

19. Lu H, Xu S. Ultrasensitive turn on molecularly imprinted fluorescence sensor for glycoprotein detection based on nanoparticles signal amplification. Sens Actuat B Chem. 2020;306:127566.

20. Luo K, Park H, Adra HJ, et al. Charge-switchable magnetic separation and characterization of food additive titanium dioxide nanoparticles from commercial food. J Hazard Mater. 2020;393:122483.

21. Shen H, Wang J, Liu H, et al. Rapid and selective detection of pathogenic bacteria in bloodstream infections with aptamer-based recognition. ACS Appl Mater Interfaces. 2016;8(30):19371-8.

22. Martynenko IV, Kusić D, Weigert F, et al. Magneto-fluorescent microbeads for bacteria detection constructed from superparamagnetic $\mathrm{Fe} 3 \mathrm{O} 4$ nanoparticles and $\mathrm{AIS} / \mathrm{ZnS}$ quantum dots. Anal Chem. 2019;91(20):12661-9.

23. Varshney M, Yang L, Su X-L, Li Y. Magnetic nanoparticle-antibody conjugates for the separation of Escherichia coli O157:H7 in ground beef. J Food Prot. 2005;68(9):1804-11.

24. Vinayaka AC, Ngo TA, Kant K, et al. Rapid detection of Salmonella enterica in food samples by a novel approach with combination of sample concentration and direct PCR. Biosens Bioelectron. 2019;129:224-30.

25. Xu H, Tang F, Dai J, Wang C, Zhou X. Ultrasensitive and rapid count of Escherichia coli using magnetic nanoparticle probe under dark-field microscope. BMC Microbiol. 2018;18(1):100.

26. Yim C, Lee H, Lee S, Jeon S. One-step immobilization of antibodies on $\mathrm{ZIF}-8 / \mathrm{Fe} 3 \mathrm{O} 4$ hybrid nanoparticles for the immunoassay of Staphylococcus aureus. RSC Adv. 2017;7(3):1418-22.

27. Yang X, Zhou X, Zhu M, Xing D. Sensitive detection of Listeria monocytogenes based on highly efficient enrichment with vancomycin-conjugated brush-like magnetic nano-platforms. Biosens Bioelectron. 2017;91:238-45.

28. Au AK, Huynh W, Horowitz LF, Folch A. 3D-printed microfluidics. Angew Chem Int Ed. 2016;55(12):3862-81.

29. Yim SJ, Ramanjaneyulu BT, Vidyacharan S, Yang YD, Kang IS, Kim D-P. Compact reaction-module on a pad for scalable flowproduction of organophosphates as drug scaffolds. Lab Chip. 2020;20(5):973-8.

30. Mairhofer J, Roppert K, Ertl P. Microfluidic systems for pathogen sensing: a review. Sensors. 2009;9(6):4804-23.

31. Lee H, Kim G, Park E, Jeon S. Lenz's law-based virtual net for detection of pathogenic bacteria from water. Anal Chem. 2019;91(24):15585-90.

32. Huang F, Zhang H, Wang L, Lai W, Lin J. A sensitive biosensor using double-layer capillary based immunomagnetic separation and invertase-nanocluster based signal amplification for rapid detection of foodborne pathogen. Biosens Bioelectron. 2018;100:583-90.

33. Hou Y, Tang W, Qi W, Guo X, Lin J. An ultrasensitive biosensor for fast detection of Salmonella using 3D magnetic grid separation and urease catalysis. Biosens Bioelectron. 2020;157:112160.

34. Yao L, Zheng L, Cai G, Wang S, Wang L, Lin J. A rapid and sensitive salmonella biosensor based on viscoelastic inertial microfluidics. Sensors. 2020;20(9):2738.
35. Lee J-H, Lee S-K, Kim J-H, Park J-H. Separation of particles with bacterial size range using the control of sheath flow ratio in spiral microfluidic channel. Sens Actuat A Phys. 2019;286:211-9.

36. Sung Y, Suk H, Sung H, Li T, Poo H, Kim M. Novel antibody/gold nanoparticle/magnetic nanoparticle nanocomposites for immunomagnetic separation and rapid colorimetric detection of Staphylococcus aureus in milk. Biosens Bioelectron. 2013;43:432-9.

37. Hwang J, Kwon D, Lee S, Jeon S. Detection of Salmonella bacteria in milk using gold-coated magnetic nanoparticle clusters and lateral flow filters. RSC Adv. 2016;6(54):48445-8.

38. Li F, et al. Biotin-exposure-based immunomagnetic separation coupled with nucleic acid lateral flow biosensor for visibly detecting viable Listeria monocytogenes. Anal Chim Acta. 2018;1017:48-56.

39. Lee $\mathrm{H}$, Han $\mathrm{H}$, Jeon $\mathrm{S}$. Baleen-mimicking virtual filters for rapid detection of pathogenic bacteria in water using magnetic nanoparticle chains and a Halbach ring. ACS Sens. 2020;5(11):3432-7.

40. Xia N, Hunt TP, Mayers BT, et al. Combined microfluidic-micromagnetic separation of living cells in continuous flow. Biomed Microdevices. 2006;8(4):299.

41. Pamme N, Wilhelm C. Continuous sorting of magnetic cells via on-chip free-flow magnetophoresis. Lab. 2006;6(8):974-80.

42. Abafogi AT, Kim J, Lee J, Mohammed MO, van Noort D, Park S. 3D-printed modular microfluidic device enabling preconcentrating bacteria and purifying bacterial DNA in blood for improving the sensitivity of molecular diagnostics. Sensors. 2020;20(4):1202.

43. Jung SH, Hahn YK, Oh S, et al. Advection flows-enhanced magnetic separation for high-throughput bacteria separation from undiluted whole blood. Small. 2018;14(34):1801731.

44. Jung T, Jung Y, Ahn J, Yang S. Continuous, rapid concentration of foodborne bacteria (Staphylococcus aureus, Salmonella typhimurium, and Listeria monocytogenes) using magnetophoresis-based microfluidic device. Food Control. 2020;114:107229.

45. Ngamsom B, Esfahani MMN, Phurimsak C, et al. Multiplex sorting of foodborne pathogens by on-chip free-flow magnetophoresis. Anal Chim Acta. 2016;918:69-76.

46. Posthuma-Trumpie GA, Korf J, van Amerongen A. Lateral flow (immuno)assay: its strengths, weaknesses, opportunities and threats. A literature survey. Anal Bioanal Chem. 2009;393(2):569-82.

47. Armbrecht L, Dincer C, Kling A, Horak J, Kieninger J, Urban G. Self-assembled magnetic bead chains for sensitivity enhancement of microfluidic electrochemical biosensor platforms. Lab Chip. 2015;15(22):4314-21.

48. Cai G, Wang S, Zheng L, Lin J. A fluidic device for immunomagnetic separation of foodborne bacteria using self-assembled magnetic nanoparticle chains. Micromachines. 2018;9(12):624.

49. Wang L, Huo X, Zheng L, et al. An ultrasensitive biosensor for colorimetric detection of Salmonella in large-volume sample using magnetic grid separation and platinum loaded zeolitic imidazolate Framework-8 nanocatalysts. Biosens Bioelectron. 2020;150:111862.

50. Nemr CR, Smith SJ, Liu W, et al. Nanoparticle-mediated capture and electrochemical detection of methicillin-resistant Staphylococcus aureus. Anal Chem. 2019;91(4):2847-53.

51. Sun X, Lei C, Guo L, Zhou Y. Separable detecting of Escherichia coli $\mathrm{O} 157 \mathrm{H}: \mathrm{H} 7$ by a giant magneto-resistance-based bio-sensing system. Sens Actuat B Chem. 2016;234:485-92.

52. Wang R, Xu Y, Sors T, Irudayaraj J, Ren W, Wang R. Impedimetric detection of bacteria by using a microfluidic chip and silver nanoparticle based signal enhancement. Microchim Acta. 2018;185(3): 184 .

53. Yoo SM, Lee SY. Optical biosensors for the detection of pathogenic microorganisms. Trends Biotechnol. 2016;34(1):7-25. 
54. Shen J, Zhou T, Huang R. Recent advances in electrochemiluminescence sensors for pathogenic bacteria detection. Micromachines. 2019;10(8):532.

55. Li Y, Cheng H, Alhalili Z, Xu G, Gao G. The progress of magnetic sensor applied in biomedicine: a review of non-invasive techniques and sensors. J Chin Chem Soc. 2021;68(2):216-27.

56. Chen C-X, Li Y-H, Zhou Y-L, et al. Rapidly detecting antibiotics with magnetic nanoparticle coated CdTe quantum dots. RSC Adv. 2020;10(4):1966-70.

57. Chang Z-M, Wang Z, Shao D, et al. Fluorescent-magnetic Janus nanorods for selective capture and rapid identification of foodborne bacteria. Sens Actuat B Chem. 2018;260:1004-11.

58. Zhang H, Yao S, Song X, et al. One-step colorimetric detection of Staphylococcus aureus based on target-induced shielding against the peroxidase mimicking activity of aptamer-functionalized goldcoated iron oxide nanocomposites. Talanta. 2021;232:122448.

59. Faridi MA, Ramachandraiah H, Banerjee I, Ardabili S, Zelenin S, Russom A. Elasto-inertial microfluidics for bacteria separation from whole blood for sepsis diagnostics. J Nanobiotechnol. 2017;15(1):3.

60. Lee W, Kwon D, Choi W, et al. 3D-printed microfluidic device for the detection of pathogenic bacteria using size-based separation in helical channel with trapezoid cross-section. Sci Rep. 2015;5(1):7717.

61. Kwon D, Joo J, Lee J, Park K-H, Jeon S. Magnetophoretic chromatography for the detection of pathogenic bacteria with the naked eye. Anal Chem. 2013;85(15):7594-8.
62. Rengaraj S, Cruz-Izquierdo Á, Scott JL, Di Lorenzo M. Impedimetric paper-based biosensor for the detection of bacterial contamination in water. Sens Actuat B Chem. 2018;265:50-8.

63. He PJW, Katis IN, Kumar AJU, et al. Laser-patterned paperbased sensors for rapid point-of-care detection and antibioticresistance testing of bacterial infections. Biosens Bioelectron. 2020;152:112008.

64. Shim W, Lee C, Kim M, Chung D. An antibody-magnetic nanoparticle conjugate-based selective filtration method for the rapid colorimetric detection of Listeria monocytogenes. Anal Methods. 2014;6(22):9129-35.

65. Shim W, Song J, Mun H, Chung D, Kim M. Rapid colorimetric detection of Salmonella typhimuriumusing a selective filtration technique combined with antibody-magnetic nanoparticle nanocomposites. Anal Bioanal Chem. 2014;406:859-66.

66. Ren W, et al. Ultrasensitive detection of microbial cells using magnetic focus enhanced lateral flow sensors. Chem Commun (Camb). 2016;52(27):4930-3.

67. Wang C, et al. Layer-by-layer assembly of magnetic-core dual quantum dot-shell nanocomposites for fluorescence lateral flow detection of bacteria. Nanoscale. 2020;12(2):795-807.

Publisher's Note Springer Nature remains neutral with regard to jurisdictional claims in published maps and institutional affiliations. 